\title{
Los restos de Cristobal Colón en Santo Domingo
}

\author{
DOI: http://dx.doi.org/10.5377/koot.v0i9.5907 \\ URI: http://hdl.handle.net/11298/437
}

\author{
Dr. Jaime Alberto López Nuila \\ Abogado e Investigador UTEC
}

\section{Resumen}

La posesión de los restos de Cristóbal Colón es una cuestión de honor y de honra para los dominicanos, que afirman disponer históricamente del cariño del descubridor, quien expresamente declaro que sus restos descansaran en el territorio dominicano.

Este hecho histórico se complica, ya que España, por su parte, siempre ha querido recuperar los restos de este audaz navegante y descubridor.

España, que históricamente había ocupado la isla, se ve enfrascada con una potencia enemiga, Francia, que asume el control de todo el territorio, hecho que complica el quehacer socioeconómico, político e histórico. No obstante, para España, siempre sigue siendo una cuestión de orgullo y dignidad, recuperar los restos de tan importante personaje para el reino y por ende para la historia.

El material bibliográfico utilizado en este artículo por el autor, es un fiel referente, con datos fidedignos sobre este tan importante hecho histórico.

Palabras clave: Colón, Cristóbal, 1451-1506 - Tumba, Antropología, Historia Conquista, República Dominicana - Descubrimiento y conquista.

\begin{abstract}
The possession of the remains of Christopher Columbus is a matter of honor for Dominicans who claim to hold historically the affection of the discoverer who specifically stated that his remains rest in the Dominican territory.

This historical fact is complicated since Spain, for its part, has always wanted to recover the remains of this audacious navigator and discoverer.

Spain, which had historically occupied the island, is involved with its nemesis in power, France, that assumes control of the entire territory and complicates socio-economic, political and historical mainstream. However, for Spain, it is always a matter of pride and dignity, to recover the remains of such an important
\end{abstract}


character for the kingdom and therefore for history.

The bibliographic material used in this article by the author, is an authentic reference, with reliable data on this important historical fact.

Keywords: Columbus Christopher, 1451-1506 - Tomb, Anthropology, History - Conquest, Dominican Republic - Discovery and conquest.

\section{Contexto Histórico. Los Viajes de Colón al Nuevo Mundo.}

La Isla de Santo Domingo ó La Española comprende el espacio territorial de la República Dominicana y Haití, es parte de las llamadas Antillas Mayores, la segunda en tamaño después de Cuba, y tiene una extensión de 77,000 $\mathrm{kms}^{2}$ correspondiendo a República Dominicana $48,442 \mathrm{Km}^{2}$ y el resto a Haití. Los límites de la isla son al norte el Océano Atlántico, al sur el Mar Caribe también conocido como Mar de las Antillas, al este el Canal de la Mona que lo separa de Puerto Rico, y al oeste, el Canal de El Viento que lo divide de las Islas de Cuba y Jamaica. ${ }^{1}$

Lo que hoy conocemos como República Dominicana ha sido llamada de forma diferente, relacionada con su condición de Isla. Antiguamente se le llamó Haití, que significaba Tierra Alta y era el nombre que le daba el indígena originario, quienes también llamaron Quisqueya a la parte situada al oriente, y Boquete o Bohio, a la que se sitúa al occidente. Dicen que Quisqueya significaba para el nativo Madre de la Tierra. Con la llegada de los europeos, con Colón a la cabeza, la isla recibe el nombre de la Hispaniola, desde el Siglo XV hasta el siglo XIX, al tiempo que también se le llamaba Santo Domingo, que gozaba cierta predilección ante sus habitantes. ${ }^{2}$

La sociedad de la República Dominicana es producto de una mezcla de al menos tres grupos sanguíneos totalmente diferentes: la indígena, nativa del territorio, el conquistador español y el negro importado como esclavo. El conquistador español se une al nativo indígena y origina el mestizo. Cuando aparece en escena el negro esclavo procedente de África, se origina el conocido como mulato. Con el transcurso del tiempo esta triple oferta racial, mezclada entre sí profusamente y sin distingos, la raza dominicana variada encuentra tipos puros y tipos mezclados.

La raza aborigen, por las enfermedades importadas por los otros dos grupos raciales, más fuertes, es prácticamente extinguida y solo los grupos de europeos y africanos pueden llamarse, después de al menos cien años, tipos puros de Santo Domingo. Como un dato estadístico de especial interés hay que saber que la mezcla de aquellos dos grupos étnicos sobrevivientes al final, producen la

1 De los Santos, Danilo. Visión General de la Historia Dominicana. Editora Cornipio $8^{\text {a }}$. Edición. Año 1978.

2 Ibid. 


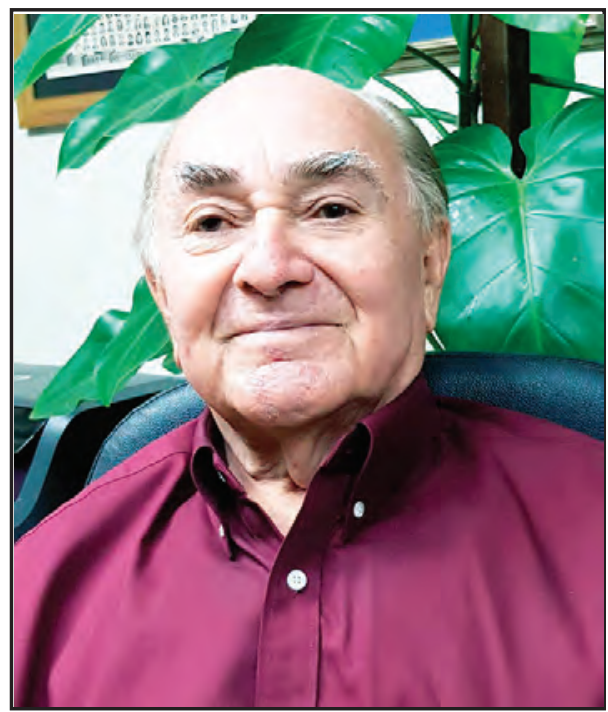

Dr. Jaime Alberto López Nuila

tipificación racial conforme al siguiente detalle: Mestizo: hijo de Blanco e indio; Mulato: hijo de negro y blanco; Grifo: hijo de indio y negro; prácticamente extinguido; Tercerón: hijo de mulato y blanco; y, el Cuarterón: si es producto de un Tercerón y un blanco. Son conocidos también los variados términos raciales que identifican después al Dominicano, así: Indio Claro, Indio Oscuro, Blanco Jojoto, Moreno, entre otros más. ${ }^{3}$

La historia de la República Dominicana se divide en tres periodos, el primero de los cuales comprende la llamada Colonización, que inicia con el suceso del Descubrimiento atribuido al Almirante Don Cristóbal Colón, ocurrido en Octubre del año 1492, seguido de la no menos importante empresa que fue la conquista de la Española. Colón es en verdad el primer historiador, por los relatos que hace, tanto en el Diario Marítimo que estaba obligado a llevar, como en sus abundantes cartas que son el medio más idóneo y disponible para comunicarse con la Capital y con sus Reyes, en aquella época. Colón describe la isla en sus aspectos geográficos, y sobre la población original de aquellos territorios, aparte de los temas que debía informar a aquella metrópoli para la administración de la isla, además de los relacionados con sus intereses personales. Fray Bartolomé de Las Casas y Gonzalo Fernández de Oviedo son parte destacada, junto a Cristóbal Colón, como fuentes directas de lo que hoy forma parte de la historiografía de la Dominicana. De Las Casas, con su reconocida obra Historia de las Indias, cuya edición publicada por la sociedad Dominicana de Bibliofilos, Editora Corripio en el año 1987 consta de tres tomos, es una obra inconmensurable, de altísimo valor; y el segundo, Don Gonzalo

3 Ibid. 
Fernández de Oviedo con su obra Historia General y Natural de las Indias, otra obra de singular interés, abundando en datos valiosos sobre la geografía de la isla, la flora y la fauna. ${ }^{4}$

El descubrimiento de América es un importante acontecimiento, tanto para el mundo de aquel siglo, como para la España de los Reyes Católicos, especialmente, que padecían de graves problemas económicos. El Genovés ofreció a la Monarquía Española los recursos para consolidar su gobierno, afirmando que navegando hacia el oeste podía llegar a la India, en breve tiempo, de tal modo que, mediante las llamadas Capitulaciones de Santa Fe, los Reyes acuerdan autorizar y sostener la empresa de Colón, prometiendo al almirante, reconocimientos y títulos en las tierras que descubriese, lo mismo que importantes beneficios en los réditos económicos de la empresa del descubrimiento.

En octubre de 1492 Colón descubre la isla que llamó La Española, porque creyó encontrar en aquellas tierras la fauna y la vegetación propia de España. Razones económicas pues, importan al europeo para iniciar aquellas expediciones marítimas, siendo el oro y las especies lo que constituía el primer objetivo de aquella aventura. Colón llegó luego de salir del Puerto de Palos y hacer escala en las Islas Canarias, al Archipiélago de las Bahamas, a la Isla llamada Guanahaní por los indígenas que allí vivían, isla que Colón bautizó con el nombre de San Salvador. Luego llegó a las de Cuba y Haití a las que colón llamó Juana y La Española. El efecto inmediato del descubrimiento de estas islas del actual Caribe, ofrece a España las lógicas posibilidades de su desarrollo económico basado en el flujo de metales preciosos y nuevos productos agrícolas como la papa, el maíz, el cacao y el tabaco. Este nuevo panorama ofrece el traslado del desarrollo comercial, del Mediterráneo como era hasta entonces, hasta el Atlántico y posibilita el fortalecimiento de las potencias políticas como Portugal, Francia, Holanda, España e Inglaterra, acérrimos rivales que disputarán el predominio marítimo y colonial. Se inicia también la esclavitud como instrumento para la conquista y posterior explotación del nuevo mundo. ${ }^{5}$

El dos de noviembre de 1493, Cristóbal Colón regresa a la Española luego de un tiempo en España. Este segundo viaje ofrece para Colón y para España, los recursos y el propósito de consolidar la conquista de aquellos territorios, y todos los nuevos que habían de ser descubiertos en este nuevo viaje. Viajan con Colón 17 navíos y 1,500 hombres, dotados de las armas y equipo necesario, y además con plantas, animales y semillas para establecer la nueva factoría y colonias como punto de inicio de la conquista. Toda la campaña del descubrimiento y la conquista posterior a la colonización del nuevo mundo, se organizan con el propósito final de búsqueda y obtención del oro.

4 Ibid.

5 Ibid. 
La administración del nuevo mundo, ampliado luego con la llegada del conquistador europeo a tierra firme en América, iniciada en Venezuela y luego al resto del continente y la creciente tensión entre Colón y sus familiares con el resto de la expedición europea, originada solo por el ánimo de lucro y enriquecimiento, motiva que al final Cristóbal Colón es despojado de sus funciones y prerrogativas en el control de la nueva Colonia, siendo incluso detenido y preso por Francisco de Bobadilla, quien había llegado de España con claras instrucciones de despojar al almirante Cristóbal Colón, de la administración y gobernación de la Isla la Española. Colón es incluso enviado de regreso a España, culminando de esta manera el importante papel de descubridor de un nuevo mundo, para beneficio de los Re yes Católicos de España y también para beneficio de la historia y desarrollo de la humanidad. ${ }^{6}$

\section{II) Muerte del Almirante. Los Viajes de sus Restos Mortales.}

Don Cristóbal Colón murió, supuestamente, el día 20 de mayo del año 1506 en Valladolid, España, en el Convento de San Francisco, siendo celebradas sus exequias en la Parroquia de Santa María de la Ciudad de Valladolid. En el año 1513, sus restos fueron trasladados al Monasterio de Cartujas de las Cuevas, en la Ciudad de Sevilla, a la Capilla de Santa Ana o Santo Cristo, y en aquella capilla fueron depositados los restos de su hijo Don Diego Colón. Como el Almirante había expresado su deseo de ser exhumado después de su muerte en la Isla de Santo Domingo, en el año 1536 los restos mortales de Cristóbal Colón, y su hijo Diego Colón, fueron entregados y trasladados a la Isla, quedando en el Monasterio de Las Cuevas de Sevilla, solamente el cuerpo de Don Bartolomé Colón.?

Hay acuerdo total entre los cronistas de que, en efecto, en el año 1536 las reliquias de Cristóbal y su hijo Diego Colón fueron trasladados de Sevilla a Santo Domingo, y depositados en el Presbiterio o Capilla Mayor de la Catedral. Aparece asimismo que según el testamento de Don Diego Colón el día 16 de marzo de 1509, clausula undécima, Diego mandó depositar en el Monasterio de Las Cuevas de Sevilla, España, el cuerpo de su padre Don Cristóbal Colón y luego en 1536 o 1539, fueron sacados de La Cuevas, y trasladados a Santo Domingo, pero no fue posible depositarlos de inmediato en el Presbiterio o Capilla Mayor de la Catedral, sino hasta el año 1541 o poco después, ya sea porque no estaban concluidos todavía los trabajos de construcción de la Catedral, o porque hasta 1541 aproximadamente, se logró vencer la oposición a aquel enterramiento por parte de los miembros del cabildo de aquella Catedral. ${ }^{8}$

Es decir que el Almirante tuvo una existencia agitada en la vida y también en la muerte, dado que, fallecido en Valladolid y enterrado en ese lugar en el

6 Ibid.

7 Tejera, Emiliano. Los restos de Colón en Santo Domingo. República Dominicana. Editorial Garcia hermanos, 1878. (primera edición). 
Convento de San Francisco en el año 1506, luego, en el año 1513, sus restos fueron trasladados al Monasterio Las Cartujas de Las Cuevas en Sevilla, para finalmente, llegar a Santo Domingo en donde fueron enterrados. Se dice que los restos del Almirante fueron sacados de las Cuevas y trasladados a República Dominicana junto a los de su hijo Diego Colón, por Doña María de Toledo. La flota donde viajó la Virreina salió de ese lugar de San Lucar de Barrameda, y llegó a Santo Domingo. Se componía de 27 buques entre los cuales estaba el San Salvador a bordo del que se transportaba el Padre Fray Bartolomé de Las Casas, según sostiene en sus notas el mencionado Don Emiliano Tejera. ${ }^{9}$

Don Emiliano Tejera sostiene, que no existiendo prueba documental fehaciente, él cree que los restos de Cristóbal Colón fueron llevados a La Española en el año 1540, año en que concluyen los trabajos de construcción de la Catedral y año en que Don Luis Colón, nieto del Almirante, llegó a Santo Domingo para tomar la Capitanía General de la Colonia. Tejera Sostiene, que la llegada del nieto del Almirante, su asunción como Capitán General y la conclusión de la obra de la Catedral de Santo Domingo, convierten el escenario ideal en aquel año 1540, la fecha probablemente más justa para cumplir los deseos del Almirante de la Mar Océano Don Cristóbal Colón, descubridor del nuevo mundo y depositarlos dentro de la tumba definitiva de sus despojos mortales.

Los cronistas del descubrimiento y colonización del nuevo mundo son unánimes en aceptar que los restos de Colón se depositaron en la Capilla Mayor o Presbiterio, pero no se señala con precisión el sitio exacto en que los restos son instalados, y tampoco informan si luego del depósito, había tenido un mausoleo, sepulcro u otro monumento para señalar tan importante deposito. La omisión señala una dificultad máxima para los historiadores, supuesto el problema de la época de aquel suceso. Don Emiliano Tejera reconoce que es de obligación aceptar que Don Cristóbal Colón no tuvo, una lápida sobre su tumba, y que si la tuvo, debió ser poco duradera. Dice que en la memoria de los historiadores dominicanos, ni en su propia memoria hubo recuerdo, de haber visto lápida alguna, lo cual le resulta muy extraño ya que en la misma Catedral se conservan los restos de Don Rodrigo de Bastidas, contemporáneo de Colón y adversario político del almirante, muerto en Santo Domingo para el año 1527 junto a algunos familiares como su esposa e hijo. ${ }^{10}$

El mismo cronista Don Emiliano Tejera en su obra Los Restos de Colón en Santo Domingo, y los Restos de Cristóbal Colón, agrega la suposición de que, al ampliar el Presbiterio de Catedral, quizá para alojar otros huéspedes distinguidos, pudo haberse movido la lápida del Almirante, luego, por negligencia o descuido, debió ser arrinconada y extraviada en forma definitiva. Tejera dice que lo mismo debió ocurrir con las de Don Diego Colón y el hijo Don Luis, Duque de Veragua.

$\begin{array}{ll}8 & \text { Ibid. } \\ 9 & \text { Ibid. } \\ 10 & \text { Ibid. }\end{array}$ 
Otro interesante relato es el que Tejera deja señalado: Que la lápida de Colón, si la hubo, debió quitarse el año 1655, fecha en la que, un día 23 de abril, la Escuadra Inglesa comandada por William Peen, atraca en la rada del Puerto de Santo Domingo. Ocurre que el Arzobispo de Santo Domingo Don Francisco Pio de Guadalupe, temiendo que el invasor inglés pudiese profanar la tumba, dispuso que la sepultura de Cristóbal Colón fuese cubierta, para que no se diese ninguna profanación ni desacato de aquel lugar. ${ }^{11}$

La vida y la muerte del Almirante, descubridor del nuevo mundo, siempre agitadas, tiene un nuevo episodio marcado ahora por la política entre potencias coloniales. El Tratado de Basilea cede a Francia la posesión de Santo Domingo, razón por la que, apresuradamente, se procede en 1795 a exhumar los restos de Colón en el Presbiterio Mayor de la Catedral Metropolitana de Santo Domingo. Don Gabriel Aristizabal, jefe local de la Armada Española, por orgullo y amor patrio, no piensa abandonar al enemigo francés, los restos mortales del Almirante y se toma el tiempo para exhumar y trasladar los restos hasta la Habana, todavía en poder de España. Colón estaba enterrado en planchas de plomo, pero sin lápida ni inscripción, y por ello, los españoles al proceder a exhumar y extraer los restos, actúan conforme la tradición oral de autoridades eclesiásticas y empleados de la Catedral, o bien de los vecinos de aquella ciudad próxima al templo, y siendo que la tradición decía, que los restos de Colón estaban depositados en el Presbiterio de la Catedral, del lado del Evangelio en el lugar donde solía colocarse el Dosel Arzobispal, así procede el encargado de aquel solemne acto. ${ }^{12}$

El Acta de Exhumación de los restos de Colón, suscrita por Don José F. Hidalgo, Escribano de la Cámara de la Real Audiencia de Santo Domingo, del día 20 de diciembre de 1795 , dice, "que se abrió una bóveda que estaba sobre el Presbiterio, al lado del Evangelio, pared principal y peana del altar mayor, y en ella se encontraron unas planchas de plomo, indicante de haber habido caja de dicho metal y pedazos de huesos de canillas, y otras varias partes de algún difunto, que se recogieron en una salvilla y toda la tierra que con ella había, que por los fragmentos con que estaba mezclada se conocía ser despojos de aquel cadáver". No se dice, reflexión de Don Emiliano Tejera, que hubiese lápida sobre esa bóveda; no se dice, que hubiese inscripción alguna; ni en lo exterior ni en las planchas encontradas en lo interior. Cómo pues, podía saberse que aquellos eran los huesos de Colón? Quién podría afirmarlo si muda estaba la piedra, mudo el metal y mudos también aquellos huesos encontrados y extraídos? En aquél día, dice don Emiliano, no se sabía que a algunas pulgadas de distancia, una de otra, había dos bóvedas, cada una de las cuales encerraba restos preciosos de dos seres estrechamente unidos en la vida y también en la muerte, Don Cristóbal Colón y don Diego Colón., su hijo.

11 Ibid.

12 Ibid. 
Así fue que, de las dos bóvedas contiguas, los españoles abrieron no la bóveda pegada al muro que era la que encerraba los restos mortales del Primer almirante, sino la que, separada de la primera por una pared de 6 centímetros de grueso, y que guardaba los restos de Diego Colón. El insigne historiador Don Emiliano Tejera, afirma, tajante, "que esa bóveda, la de Diego Colón es la que se identifica claramente con la designación hecha por el Escribano José F. Hidalgo, que elaboró el Acta de Exhumación y que señala entre la pared principal y la peana del Altar Mayor, ambas bóvedas están sobre el Presbiterio, ambas del lado del Evangelio", "pero la que contiene los restos de colón, estaba y está, pegada a la pared". ${ }^{13}$

\section{III) Controversia sobre la Identidad de los Restos del Almirante.}

El celo patriótico ha generado por mucho tiempo, una afanosa disputa entre dos países, que sin duda, amaron siempre el recuerdo del descubridor del nuevo mundo: España y República Dominicana. La voluntad expresa del Almirante de descansar en suelo de la Hispaniola, hoy República Dominicana, ha determinado que ese pueblo niegue desde siempre que los despojos mortales trasladados a España, con tránsito en la Habana Cuba, en el año 1795 sean los de Cristóbal colón, efecto obligado por el Tratado de Basilea entre España y Francia, que entrega a este último país la posesión de Santo Domingo. La presurosa exhumación de los supuestos despojos mortales de Colón, hace incurrir a España en el histórico error de confundir, según lo ha dejado señalado Don Emiliano Tejera, la tumba de Diego Colón con la de su padre Cristóbal. La parte dominicana ha defendido con mucha sabiduría, su tesis de la equivocada exhumación, como lo declara, repito, el historiador dominicano citado ampliamente en este relato.

No hubo jamás retroceso en las posiciones antagónicas de España y de República Dominicana; y firmemente creo que no las habrá nunca. Quizá por esa realidad la parte española recurrió años atrás al expediente científico de realizar prueba de ADN, que estudia la identidad de las personas, haciendo una propuesta a la Universidad de Granada, para que el investigador genético Don José Antonio Lorente Acosta, colaborará a resolver ese delicado conflicto, utilizando las técnicas modernas de identificación genética. El punto de partida era que a la fecha en el año 2002 había dos lugares para ubicar la tumba de Cristóbal Colón. La solicitud tenía relación con la próxima celebración de los 500 años de su muerte. El grupo que se formó para hacer la investigación profesó su único propósito de encontrar la verdad histórica, y solo como tributo a la ciencia misma, alejado, de cualquier interés o propósito patriótico. ${ }^{14}$

13 Ibid.

14 Celso García De La Riega. Estudio ADN de Cristóbal Colón y su posible origen. Tomado de internet 24 de febrero de $2018 \mathrm{https} / / /$ celsogarciadelariega.wordpress.com/tag/estudio-adnde-cristobal-colon/ 
El informe señaló que se han comparado restos humanos que contienen la tumba de la Catedral de Sevilla- supuestamente del Almirante-- con los restos de su hermano menor Bartolomé Colón, enterrado en Las Cartujas, Sevilla.

La comisión de investigación formada luego de recoger los restos de Colón el 23 de mayo del año 2005, procede a su estudio, por parte del laboratorio de Antropología Física de la Universidad de Granada, y produce la conclusión de que se puede confirmar que en la tumba de Sevilla, en efecto, se encuentran depositados los restos del Almirante, después de obtener un resultado positivo, tras la comparación del ADN de ambos restos. La conclusión incluye además, el aserto de que los huesos analizados tienen una edad de 500 años, la afirmación que ambos restos pertenecen a personas que son hijos de la misma madre, concluyendo así que la sepultura de Sevilla es auténtica. Hay también consideraciones muy importantes en el contexto del informe, como que es probablemente seguro que tanto los restos de Santo Domingo como los examinados de Sevilla contienen restos auténticos de Cristóbal Colón. Debe aclararse al respecto, que los restos de Santo Domingo no han sido parte del material sometido al examen y análisis científico de la comisión investigadora.

Además y para terminar esta breve información sobre la autenticidad de los restos de colón depositados en Sevilla, está el hecho reconocido de que los restos de Sevilla están incompletos, ya que falta el cráneo y la mandíbula, y por tanto, los mismos son ciertamente muy escasos. Del resultado de la investigación científica antes señalada puedo dejar asentado, por tanto, que no obstante la afirmación positiva del informe del ADN, de los restos de Sevilla, la controversia en orden de la legitimidad y autenticidad, en comparación con los restos que se conservan en la catedral de Santo Domingo, seguirá pendiente. La alternativa científica no estará concluida mientras no se practiquen iguales pruebas con los despojos que están en la Isla, lo que requerirá el consentimiento y la participación Dominicana, para iniciar esa investigación.

\section{Referentes bibliográficos}

García de la Riega, C. (2018). Estudio ADN de Cristóbal Colón y su posible origen. Recuperado de https://celsogarciadelariega.wordpress.com/tag/ estudio-adn-de-ccristobal-colon/

Peguero, V., \& De los Santos, D. (1978). Visión general de la historia Dominicana (8va ed.). Santo Domingo: Editora Cornipio.

Tejera, E. (1926). Los restos de Colón en Santo Domingo y los dos restos de Cristóbal Colón. (4ta ed.). Santo Domingo: J. R. Vda. García. 